\title{
Ability of the rumen ciliate protozoon Eudiplodinium maggii to digest and ferment microcrystalline cellulose
}

\author{
T Michalowski \\ The Kielanowski Institute of Animal Physiology and Nutrition, 05-110 Jablonna, Poland
}

The rumen ciliate Eudiplodinium maggii was separated and cultured in vitro in a species culture, as described earlier (Michalowski et al, 1991, Acta Protozool, 30, 115-120). The protozoon readily engulfed particles of microcrystalline cellulose "Sigmacell 20 ". Microscopic observations of the cultured ciliate showed a gradual disappearance of engulfed particles from cells over a $24 \mathrm{~h}$ period.

The $10000 \mathrm{~g}$ supernatant fraction of broken cells hydrolysed microcrystalline cellulose to reducing sugars with a $\mathrm{pH}$ optimum of 5.5 . Polyacrylamide gel electrophoresis (PAGE) of this fraction on gels containing carboxy- methylcellulose (CMC) followed by incubation of the gels in PC buffer ( $\mathrm{pH} 5.5)$ and staining with 2,3,5 triphenyltetrazolium chloride (Gabriel and Wang, 1969, Anal Biochem, 27, 545-555) showed endoglucanase type activity in the preparation.

The incubation of ciliates, free of bacteria, in the presence of microcrystalline cellulose and chloramphenicol resulted in an increase in the concentration of volatile fatty acids in the incubation medium. Acetic acid followed by butyric acid were the main acids produced by protozoa. 